\title{
4 Evolution of dosage compensation does not depend on genomic background
}

5

6

7 Michail Rovatsos*, Lukáš Kratochvíl

8

9

10 Department of Ecology, Faculty of Science, Charles University, Prague, 12844, Czech

11 Republic

12

13 *Corresponding author: Email mirovatsos@gmail.com 


\section{Abstract}

15 Organisms evolved various mechanisms to cope with the differences in the gene copy numbers between sexes caused by degeneration of $\mathrm{Y}$ and $\mathrm{W}$ sex chromosomes. Complete

17 dosage compensation or at least expression balance between sexes was reported predominantly in $\mathrm{XX} / \mathrm{XY}$, but rarely in $\mathrm{ZZ/ZW}$ systems. However, this often-reported pattern is based on comparisons of lineages where sex chromosomes evolved from non-homologous genomic regions, potentially differing in sensitivity to differences in gene copy numbers. Here we document that two reptilian lineages (XX/XY iguanas and ZZ/ZW softshell turtles), which independently co-opted the same ancestral genomic region for the function of sex chromosomes, evolved different gene dose regulatory mechanisms. The independent cooption of the same genomic region for the role of sex chromosome as in the iguanas and the softshell turtles offers a great opportunity for testing evolutionary scenarios on the sex chromosome evolution under the explicit control for the genomic background and for gene identity. We showed that the parallel loss of functional genes from the Y chromosome of the green anole and the $\mathrm{W}$ chromosome of the Florida softshell turtle led to different dosage compensation mechanisms. Our approach controlling for genetic background thus does not support that the variability in the regulation of the gene dose differences is a consequence of ancestral autosomal gene content.

\section{Keywords}

34 Anolis, dosage compensation, gene expression, sex chromosomes, softshell turtles, 


\section{Introduction}

Sex chromosomes evolve from a pair of autosomes, where one chromosome acquires a sexdetermining locus. The regions around this sex-determining locus often stop recombination with their respective homologous regions on $\mathrm{X}$ or $\mathrm{Z}$ chromosomes (Muller, 1918; Ohno, 1967; reviewed in Charlesworth, Charlesworth, \& Marais. 2005), potentially due to sexually antagonistic selection, which leads to the accumulation of alleles beneficial for one sex but detrimental to the other in the vicinity of the sex-determining locus. Over time, the cessation of recombination triggers changes mainly in the $\mathrm{Y}$ and $\mathrm{W}$ chromosomes, including the accumulation of deleterious mutations and extensive degradation of the gene content. Thus, the differentiation of sex chromosomes leads to unequal numbers of functional copies of many genes between the sexes. These differences have to be in some way handled at the cellular level, as the protein production in a cell is generally affected by the number of transcribed gene copies, and cell physiology and differentiation require proper stoichiometric ratios of interacting proteins (Birchler, Riddle, Auger, \& Veitia, 2005; Zhag \& Oliver, 2007; Birchler, 2014; Dürrbaum \& Storchová 2016). Different lineages evolved distinct mechanisms to cope with the gene copy disequilibrium. Some lineages evolved dosage compensation, the epigenetic mechanism which restores the expression of the $\mathrm{X}$ - or $\mathrm{Z}$ specific genes in the heterogametic sex to the ancestral autosomal levels (Muller, 1918; Ohno, 1967; Brockdorff \& Turner, 2015).

Despite the common features of the differentiation process of sex chromosomes, it was suggested that there is a dichotomy in the gene dose regulatory mechanisms between male heterogamety (XX/XY) and female heterogamety (ZZ/ZW systems). Complete dosage compensation or at least parity in the expression of the X- or Z- specific genes between sexes (this parity is also referred to as "dosage balance" in the expression levels by some authors, e.g. Gu \& Walters, 2017) was often found in lineages with XX/XY sex chromosomes. The 
61

term dosage balance refers to the situation where the expression of the $\mathrm{Z} / \mathrm{X}$-specific gene is equal between the two sexes, regardless of the ancestral expression level of the same gene when it was autosomal (Gu \& Walters, 2017). Such mechanisms have been documented in several insect lineages, nematode worms, therian mammals and the green anole (reviewed in Gu \& Walters, 2017). However, lack of dosage balance in the expression of X-specific genes was found in three lineages with male heterogamety: the three-spined stickleback, the platypus and the brown basilisk (Gu \& Walters, 2017; Acosta et al., 2019; Nielsen et al., 2019). A lack of dosage balance seems to be common in lineages with female heterogamety, where it was documented in parasitic bloodflukes, tonguefish, caenophidian snakes, the Komodo dragon and birds (Mank, 2009; Vicoso, Emerson, Zektser, Mahajan, \& Bachtrog, 2013; Gu \& Walters 2017; Picard et al., 2018; Rovatsos, Rehák, Velenský, \& Kratochvíl, 2019). The single exception is lepidopteran insects, where dosage balance was found, but the level of expression of Z-specific genes is likely lower than the ancestral state (Huylmans, Macon, \& Vicoso, 2017). However, from the above list of taxa it is evident that our knowledge of gene dose regulatory mechanisms is limited to comparisons of a small number of lineages with highly dissimilar general biology and complexity of body plans and genomes. Moreover, the comparison between differentiated sex chromosomes under male and female heterogamety can be strongly confounded by the non-homology of sex-linked genes among these lineages and consequently, the different tolerance to copy variation of dosage sensitive genes, located in sex chromosomes. By a dosage sensitive gene, we refer to any gene where a change in gene dosage (e.g. copy number variation) causes a phenotypic effect, no matter the precise mechanism of dosage sensitivity (reviewed e.g. in Rice \& McLysaght, 2017; Pessia, Engelstädter, \& Marais, 2014; Zimmer, Harrison, Dessimoz, \& Mank, 2016).

Our study suggests a solution to these problems. We compared the gene dose regulatory mechanism in two reptile lineages (i) with opposite heterogamety and (ii) ancient highly 
86

differentiated sex chromosomes, which (iii) independently evolved from the same genomic region: the iguanian green anole (Anolis carolinensis) with male heterogamety (Alföldi et al., 2011) and the Florida softshell turtle (Apalone ferox) with female heterogamety (Rovatsos, Praschag, Fritz, \& Kratochvíl, 2017). Both lineages co-opted the same genomic region for the function of sex chromosomes containing genes with orthologs linked to chicken (GGA) chromosome 15 (GGA15) (Alföldi et al., 2011; Rovatsos et al., 2017; Marin et al., 2017). Twelve families of iguanas including anoles share the same X-specific gene content, which documents that differentiated $\mathrm{XX} / \mathrm{XY}$ chromosomes homologous to those of the green anole were present already in the common ancestor of iguanas living at least $c$. 70-95 million years ago (Rovatsos, Pokorná, Altmanová, \& Kratochvíl, 2014a; Altmanová, et al., 2018). In the softshell turtles, the differentiated ZZ/ZW sex chromosomes are stable and can be traced back to the last common ancestor of the extant species, as 10 trionychid species covering the phylogenetic diversity of the family share the same Z-specific genes (Rovatsos et al., 2017). This evidence suggests that trionychid sex chromosomes are likely older than 120 million years (Rovatsos et al., 2017). The long-term stability of sex chromosomes in both lineages should have assured sufficient time for the emergence of an optimal gene dose regulatory mechanism.

The gene content of the $\mathrm{X}$ chromosome in the green anole has been extensively identified (Alföldi et al., 2011; Rovatsos, Altmanová, Johnson Pokorná, \& Kratochvíl, 2014b; Marin et al., 2017), the Y chromosome is highly degenerated and the complete dosage compensation was recently reported (Marin et al., 2017; Rupp et al., 2017). The dosage compensation in the green anole is reached by up-regulation of genes linked to $\mathrm{X}$ chromosome in males. This careful regulation suggests that the genes linked to the $\mathrm{X}$ chromosome should be highly dosage sensitive. We therefore predicted that we would find a similar mechanism in the turtle, where the $\mathrm{Z}$ chromosome was derived from the same 
ancestral autosome and the $\mathrm{W}$ is also highly degenerated (Rovatsos et al., 2017). Here, we test this hypothesis by determining the Z-specific genes and the sexual differences in their expression in the Florida softshell turtle A. ferox and by comparing the expression pattern of the same orthologous genes which are X-specific in the anole and at the same time Z-specific in the turtle.

\section{Material and methods}

\section{Studied material}

Two males and two females of A. ferox were obtained from a pet shop in order to collect blood samples for genetic and genomic analyses. Genomic DNA was extracted from all samples using the DNeasy Blood and Tissue Kit (Qiagen, Germany). Total RNA was extracted using TRIzol reagent (Invitrogen, Carlsbad, CA, USA) according to the manufacturer's protocol.

\section{Illumina sequencing (DNA-seq, mRNA-seq) and bioinformatic analyses}

Genomic DNA from one male and one female of A. ferox were sequenced at high coverage (approx. 50x) by Novogene (Cambridge, UK) in Illumina HiSeq2500 platform, with 150 base pairs (bp) pair-end option (DNA-seq). Libraries from total RNA of two males and two females of A. ferox were constructed by GeneCore (EMBL, Heidelberg, Germany) (mRNAseq). The barcoded stranded mRNA-sequencing libraries were prepared using the Illumina TruSeq mRNA v2 sample preparation kit (Illumina, San Diego, CA, USA) with poly-A mRNA enrichment, implemented in the liquid handling robot Beckman FXP2. 84 bp fragments were sequenced unidirectionally in the pooled libraries using the Illumina NextSeq 500 platform. The raw Illumina reads from both DNA-seq and mRNA-seq of all individuals 
are deposited in Genbank (BioProject PRJNA608206, accession numbers SRR11149095SRR11149100).

Adapters and low-quality bases from raw reads were trimmed by Trimmomatic

utility with default parameters. Reads shorter than 50 bp were removed, resulting in the final dataset of 658-731 million reads per specimen for the DNA-seq and 35-78 million reads per specimen for the mRNA-seq. Trimmed reads were checked in FASTQC (Andrews 2010) and MULTIQC (Ewels, Magnusson, Lundin, \& Käller, 2016).

In ZZ/ZW sex determination systems with a highly degenerated $\mathrm{W}$ chromosome, Zspecific genes have half copy numbers in the genomes of $\mathrm{ZW}$ females in comparison to $\mathrm{ZZ}$ males. These differences in the copy numbers of Z-specific genes between sexes are detected by the differences in coverage of the reads from DNA sequencing in Illumina HiSeq platform (e.g. Vicoso et al., 2013; Picard et al., 2018). Z-specific loci are expected to have half read coverage in $\mathrm{ZW}$ females in comparison to $\mathrm{ZZ}$ males, while autosomal and pseudoautosomal loci should have equal read coverage in both sexes. We used this approach for identification of Z-specific genes in A. ferox. Trimmed DNA-seq reads from a male and a female were independently mapped to a reference dataset of 174,456 exonic sequences previously published in the genome project of the Chinese softshell turtle, Pelodiscus sinensis, the closest related species to A. ferox with a well-annotated genome (Wang et al., 2013) using Geneious v. R7.1 (for parameters see Table S1). The read coverage of each exon was extracted and the average coverage for an individual gene was calculated in each specimen. We normalized the coverage of each gene for the total number of assembled reads per specimen (see Vicoso et 
160 (Grabherr et al., 2011), resulting to 165,925 putative transcripts. The assembled transcripts

were compared to the reference transcriptome of Pelodiscus sinensis (Wang et al., 2013)

sinensis were used as the reference transcriptome for further analyses. The Illumina reads

from all individuals were mapped independently to this reference transcriptome using

Geneious v. R7.1 (for parameters see Table S1). We filtered out all loci not expressed in at

least one individual or with transcript length less than $500 \mathrm{bp}$. To avoid pseudoreplications at the gene level, the subsequent analyses were done using just the longest transcript per gene.

We assigned genes to putative syntenic blocks according to chromosome position of their orthologous genes in the chicken genome (http://www.ensembl.org). This procedure is substantiated by the high level of conservation in gene synteny between chicken and turtles (O'Connor et al., 2018). Furthermore, the chicken has one of the best assembled genomes among sauropsids at the chromosome level, facilitating cross-species comparisons. We used this procedure to test whether the region containing Z-specific genes in A. ferox is indeed syntenic to GGA15 and thus to the $\mathrm{X}$ chromosome of the green anole as previously stated (Rovatsos et al., 2017).

Validation of Z-specific gene identification by $q P C R$

179 We used qPCR for estimation of the difference in gene copy number between male and female genomes in A. ferox to validate Z-specificity in selected genes and thus to further 
184 (adarb2, mos, $\operatorname{rag} 1$ ) genes were previously published for the trionychid turtles by Rovatsos et

185 al. (2017). For the validation we used DNA isolated from three males and three females of $A$.

186 ferox.

187

Test of dosage balance in the expression of Z-specific genes in $\mathrm{A}$. ferox and direct

comparison to A. carolinensis

The RPKM expression values were independently calculated for each transcript with average

read coverage higher than 10 in each specimen, resulting in a final dataset with expression

data from 5,616 genes (Table S2). Subsequently, we computed the average sex-specific

RPKMs for each transcript as the mean value from the two females and two males,

comparing the female to male ratios in RPKM between Z-specific genes and other genes by

Mann-Whitney $U$ test. We $\log _{2}$-transformed the ratios to improve the symmetry of the

distribution of ratios. The non-parametric test was used as Kolmogorov-Smirnov test showed that the data significantly deviate from normality $(\mathrm{p}<0.01)$. Genes with female to male ratio above 2.0 (in total less than $0.8 \%$ of genes) were excluded from the analyses as they likely interpretation. genes of the turtle and of their X-specific orthologs in the green anole directly on a gene-bygene basis. We identified X-specific orthologs of the A. ferox Z-specific genes with expression data in the green anole in the data from Rupp et al. (2017). We compared the 
Single copy genes linked to the Z-specific region are hemizygous and their transcripts

thus should not have any SNPs in females. We utilize these characteristics in combination

with information on read coverage in the male and female genomes to identify Z-specific

ratio in read coverage depth lower than 0.7 .

\section{Results}

The comparative read coverage analysis was performed in 19,151 genes of A. ferox, revealing 568 genes with female to male ratio for read coverage less than 0.7 , corresponding to Zgenes with known chromosomal position of orthologs in chicken genome. Notably, 220 out of 245 potential Z-specific genes of A. ferox have orthologs linked to GGA15, while the remaining 25 genes have orthologs scattered to 16 chicken chromosomes (Table S2). We validated sexual differences in gene copy numbers in two identified Z-specific genes by qPCR, applied to male and female genomic DNA as a template. qPCR revealed the expected pattern of approximately half the number of copies in the female genome in comparison to the male genome in all tested Z-specific genes and equal gene copy number in the control autosomal genes (Fig. S1). These results corroborate that the syntenic block homologous to GGA15 is a part of the $\mathrm{Z}$ chromosome in A. ferox as previously documented by physical gene mapping in the Chinese softshell turtle, Pelodiscus sinensis (Kawagoshi, Uno, Matsubara, Matsuda, \& Nishida, 2009) and validated in 10 species of softshell turtles by the comparison of gene copy numbers between male and female genomes (Rovatsos et al., 2017). The analysis of the female to male ratios in DNA-seq read coverage uncovered that not all genes 
32 genes with orthologs linked to GGA15 show female to male ratio in read coverage higher than 0.7 , corresponding to their autosomal or pseudoautosomal position, or to poorly differentiated $\mathrm{Z}$ - and $\mathrm{W}$-specific alleles in the non-recombining region of the turtle $\mathrm{Z}$ and $\mathrm{W}$ chromosomes (Table S2).

To study whether gene expression is equal in both sexes in the turtle, we analysed our candidate Z-linked genes that had both female to male ratio in read coverage $<0.7$. This yielded a total of 118 candidate Z-specific genes in our mRNA-seq dataset. Notably, 102 of them have orthologs on GGA15, which represents $93 \%$ of the candidate Z-linked genes with known chromosomal position of orthologs in chicken genome. The female to male ratios in RPKM differ highly between these candidate Z-specific genes and autosomal and psedoautosomal genes (Mann-Whitney $U$ test: $U=55,845, \mathrm{p}<0.0001, \mathrm{n}=5,575$ ), with the median female to male ratio in the expression level being about half of the median of the other genes in our mRNA-seq dataset (Fig. 2). We conclude that there is no dosage balance in the softshell turtle in the Z-specific genes. Expression data were available for 45 orthologues of these genes that show X-specificity in A. carolinensis (Rovatsos et al., 2014a,b; Marin et al., 2017; Rupp et al., 2017) (Table S3). Wilcoxon signed-ranks test revealed that these genes have significantly higher female to male ratios in the anole in comparison to the Florida softshell turtle $(\mathrm{Z}=6.21, \mathrm{p}<0.0001, \mathrm{n}=51)$. They are expressed at similar levels in both sexes in the green anole (Fig. 3). The results stayed the same even when a more conservative criterion, i.e. to consider as Z-specific only the genes without SNPs in the turtle, was applied.

\section{Discussion}

Contrary to our expectations, the sex-specific transcription of the orthologous genes which are $\mathrm{X}$-specific in the green anole and at the same time Z-specific in the softshell turtle differ between the species. The $\mathrm{X}$-specific genes are dosage compensated in the anole, but the 
expression of the same genes when Z-specific is not equalized between sexes in the turtle (Figs. 2,3). We can thus conclude that the loss of the same functional genes from the $\mathrm{Y}$ chromosome of the green anole and the W chromosome of the Florida softshell turtle led to different dosage compensation mechanisms. Our approach controlling for genomic background and gene identity thus shows that the regulation of the gene dose differences is not a consequence of the ancestral gene content of the genomic region now playing the role of sex chromosomes. Moreover, the comparison of the sex-specific expression of the orthologous genes between the turtle and the anole suggests that the dosage compensation of the X-specific genes in the anole does not reflect their sensitivity to gene copy number variation. Orthologs of the dosage-sensitive genes should hence be compensated in the turtle as well, or they should stay in the poorly differentiated regions of the sex chromosomes or be translocated to autosomes. Alternatively, genes linked to sex chromosomes in the anole and the turtle could theoretically change sensitivity to copy number variation during evolution, or sensitive to copy number variation of a gene can be context-dependent (see Deutschbacher et al. 2005; Morrill and Amon 2019). Nevertheless, considering that gene function and expression are generally conserved across vertebrates (e.g. Chan et al. 2009), an hypothetical scenario of mass swift of dose sensitivity seems less likely to explain the differences in gene dose regulation between the Z-specific genes of the green anole and the Z-specific genes of the Florida softshell turtle.

The difference between the anole and the Florida softshell turtle in the dosage compensation mechanisms is in agreement with the often-reported differences between male and female heterogamety. The reasons why these two systems should differ in the dosage compensation mechanisms are not clear and several processes potentially responsible for this dichotomy were suggested (Vicoso and Bachtrog 2009; Mank et al. 2010; Wilson Sayres and Makova 2011; Naurin et al. 2012; Mank 2013; Mullon et al. 2015). Recently, several 
exceptions from this pattern were reported and after these additions, lineages with male

heterogamety are not significantly more likely to possess dosage balance between sexes in the

expression of genes linked to sex chromosomes than lineages with female heterogamety

(reviewed in Rovatsos et al. 2020).

We hypothesized that the evolution of dosage compensation mechanism might reflect

to some extent differences in the function of sex-determining genes. These genes principally

work in two ways: sex determination might be controlled either by the copy number of $\mathrm{X}$ or

Z-linked loci per cell (i.e. gene dosage), or by the presence of a dominant $\mathrm{W}$ or Y locus in the

genome (Clinton, 1998). The dosage-dependent sex determination can work only in the

absence of a mechanism equalizing the expression of the sex-determining locus between

sexes, at least in the time when its expression is crucial for sex determination. In contrast, a

chromosome-wide regulatory mechanism of the expression of X-and Z-linked genes leading

to dosage balance such as heterochromatinization of a single $\mathrm{X}$ copy per cell in female mammals (Brockdorff \& Turner, 2015), is compatible with the sex determination based on a dominant factor on $\mathrm{Y}$ and $\mathrm{W}$ chromosomes (e.g. sry gene in viviparous mammals) as well. In support, both studied lineages with female heterogamety likely relying on the dosagedependent mechanism, i.e. birds and caenophidian snakes (Smith et al., 2009; Rovatsos et al., 2018), do not have dosage balance in the expression of Z-specific genes (Ellegren, 2002; Vicoso et al., 2013).

At first sight, two model organisms, the fruit fly Drosophila melanogaster and the nematode worm Caenorhabditis elegans, represent a contradictory case, since their sex determination primarily relies on the number of copies of the $\mathrm{X}$ chromosome, but at the same time they have global dosage compensation achieved by upregulation of the expression of a 
mechanisms based on copy numbers (Baker and Belote 1983; Deng et al. 2011; Zanetti and Puoti 2013). These cases illustrate that detailed knowledge on molecular machinery and timing of particular steps will often be needed for testing mechanistic hypothesis on the evolution of gene dose regulatory mechanisms. Currently, our knowledge on the identity and function of sex determining loci is sporadic and restricted mainly to model organisms (Bachtrog et al., 2014; Pan et al., 2017), but we expect that our hypothesis can be tested in future when more evidence will be accumulated. Based on our hypothesis, the presence of dosage-sensitive mechanism of sex determination is more likely in the softshell turtle.

To sum up, we introduce that independent co-option of the same genomic region for the role of sex chromosome, as seen in the iguanas and the softshell turtles, offers a great opportunity for testing evolutionary scenarios on the sex chromosome evolution under the explicit control for the genomic background. Among amniotes, more lineages than the iguanas and the softshell turtles co-opted the same syntenic block for sex chromosomes, as shown for instance by our ongoing research on lacertid lizards and geckos (ZZ/ZW) and therian mammals (XX/XY) (Rovatsos et al. 2016a; 2016b). Future studies should further utilize these excellent systems to explore the convergent/divergent evolution of sex chromosomes.

\section{Data access}

The raw Illumina reads from DNA-seq and mRNA-seq of all studied individuals are deposited into the NCBI BioProject database with ID PRJNA608206 (accession numbers SRR11149095-SRR11149100).

\section{Acknowledgements}


332 Four anonymous reviewers and the editors made excellent comments to the former version of

333 the manuscript. We are grateful to Vladimír Beneš (Genecore, Heidelberg) for valuable 334 consulting on NGS sequencing, experimental design and data analysis. Computational 335 resources were provided by the CESNET LM2015042 and the CERIT Scientific Cloud 336 LM2015085 under the project "Projects of Large Research, Development, and Innovations 337 Infrastructures". This study was supported by Czech Science Foundation (project No. 17338 22604S). Internal support was provided by Charles University research project 339 PRIMUS/SCI/46 and Charles University Research Centre program (204069).

\section{References}

342 Acosta, A., Suárez-Varón, G., Rodríguez-Miranda, L. A., Lira-Noriega, A., Aguilar-Gómez, D., Gutiérrez-Mariscal, M, ... Cortez, D. (2019). Corytophanids replaced the pleurodont XY 344 system with a new pair of XY chromosomes. Genome Biology and Evolution, 11, 2666-2677. 345 Alföldi, J., Di Palma, F., Grabherr, M., Williams, C., Kong, L., Mauceli, E, ... Lindblad-Toh, 346 K. (2011). The genome of the green anole lizard and a comparative analysis with birds and 347 mammals. Nature, 477, 587-591.

348 Altmanová, M., Rovatsos, M., Johnson Pokorná, M., Veselý, M., Wagner, F., \& Kratochvíl, 349 L. (2018). All iguana families with the exception of basilisks share sex chromosomes. 350 Zoology, 126, 98-102.

351 Altschul, S. F, Gish, W., Miller, W., Myers, E. W., \& Lipman, D. J. (1990). Basic local 352 alignment search tool. Journal of Molecular Biology, 215, 403-410.

353 Andrews, S. (2010). FastQC: A quality control tool for high throughput sequence data. 354 Available: http://www.bioinformatics.babraham.ac.uk

355 Baker, B. S., \& Belote J. M. (1983). Sex determination and dosage compensation in 356 Drosophila melanogaster. Annual Review of Genetics, 17, 345-393. 
357 Bachtrog, D., Mank, J. E., Peichel, C. L., Kirkpatrick, M., Otto, S., Ashman, T. L., ... The

358 Tree of Sex Consortium. (2014). Sex determination: why so many ways of doing it? PLoS

359 Biology, 12, e1001899.

360 Birchler, J. A. (2014). Facts and artifacts in studies of gene expression in aneuploids and sex

361 chromosomes. Chromosoma, 123, 459-469.

362 Birchler, J. A., Riddle, N. C., Auger, D. L., \& Veitia, R. A. (2005). Dosage balance in gene 363 regulation: biological implications. Trends in Genetics, 21, 219-226.

364 Bolger, A. M., Lohse, M., \& Usadel, B. (2014). Trimmomatic: a flexible trimmer for Illumina sequence data. Bioinformatics, 30, 2114-2120.

366 Brockdorff, T., \& Turner, B. M. (2015). Dosage compensation in mammals. Cold Spring 367 Harbour Perspectives in Biology, 7, a019406.

368 Chan, E. T, Quon, G. T., Chua, G., Babak, T., Trochesset, M., Zirngibl, R. A., Aubin, J., 369 Ratcliffe, M. J., Wilde, A., Brudno, M., Morris, Q. D., \& Hughes, T. R. (2009). Conservation 370 of core gene expression in vertebrate tissues. Journal of Biology 16: 33.

371 Charlesworth, D., Charlesworth, B., \& Marais, G. (2005). Steps in the evolution of 372 heteromorphic sex chromosomes. Heredity, 95, 118-128.

373 Clinton, M. (1998). Sex determination and gonadal development: a bird's eye view. Journal of 374 Experimental Zoology, 281, 457-465.

375 Deng, X., Hiatt, J. B., Nguyen, D. K., Ercan, S., Sturgill, D., Hillier, W. L., Schlesinger, F., 376 Davis, C. A., Reinke, V. J., \& Gingeras, T. R. (2011). Evidence for compensatory 377 upregulation of expressed X-linked genes in mammals, Caenorhabditis elegans and Drosophila melanogaster. Nature Genetics 43, 1179-1185.

379 Deutschbauer A. M., Jaramillo D. F, Proctor M., Kumm J., Hillenmeyer M. E., Davis R. W., 380 Nislow C., \& Giaever G. (2005). Mechanisms of haploinsufficiency revealed by genome-wide 381 profiling in yeast. Genetics, 169, 1915-1925. 
382 Dürrbaum, M., \& Storchová, Z. (2016). Effects of aneuploidy on gene expression:

383 implications for cancer. FEBS Journal, 283, 791-802.

384 Ellegren, H. 2002. Dosage compensation: do birds do it as well? Trends in Genetics, 18, 2538528.

386 Ewels, P., Magnusson, M., Lundin, S., \& Käller, M. (2016). MultiQC: summarize analysis 387 results for multiple tools and samples in a single report. Bioinformatics, 32, 3047-3048.

388 Grabherr, M. G., Haas, B. J., Yassour, M., Levin, J. Z., Thompson, D. A., Amit, I., ... Regev, 389 A. (2011). Full-length transcriptome assembly from RNA-Seq data without a reference 390 genome. Nature Biotechnology, 29, 644-652.

391 Gu, L., \& Walters, J. R. (2017). Evolution of sex chromosome dosage compensation in 392 animals: a beautiful theory, undermined by facts and bedeviled by details. Genome Biology and Evolution, 9, 2461-2476.

394 Huylmans, A. K., Macon, A., \& Vicoso, B. (2017). Global dosage compensation is ubiquitous 395 in Lepidoptera, but counteracted by the masculinization of the $\mathrm{Z}$ chromosome. Molecular 396 Biology and Evolution, 34, 2637-2649.

397 Kawagoshi, T., Uno, Y., Matsubara, K., Matsuda, Y., \& Nishida, C. (2009). The ZW micro398 sex chromosomes of the Chinese soft-shelled turtle (Pelodiscus sinensis, Trionychidae, 399 Testudines) have the same origin as chicken chromosome 15. Cytogenetic and Genome 400 Research, 125, 125-131.

401 Kearse, M., Moir, R., Wilson, A., Stones-Havas, S., Cheung, M., Sturrock, S., ... Drummond, 402 A. (2012). Geneious Basic: An integrated and extendable desktop software platform for the 403 organization and analysis of sequence data. Bioinformatics, 28, 1647-1649.

404 Mank, J. E. (2009). The W, X, Y and Z of sex-chromosome dosage compensation. Trends in 405 Genetics, 25, 226-233. 
406 Mank, J. E. (2013). Sex chromosome dosage compensation: definitely not for everyone.

407 Trends in Genetics, 29, 677-683.

408 Mank, J. E., Vicoso, B., Berlin, S., \& Charlesworth, B. (2010). Effective population size and

409 the faster-X effect: empirical results and their interpretation. Evolution, 64, 663-674.

410 Marin, R., Cortez, D., Lamanna, F., Pradeepa, M. M., Leushkin, E., Julien, P., ... Kaessmann,

411 H. (2017). Convergent origination of a Drosophila-like dosage compensation mechanism in a

412 reptile lineage. Genome Research, 27, 1974-1987.

413 Morrill S. A., \& Amon A. (2019). Why haploinsufficiency persists. Proceedings of the

$414 \quad$ National Academy of Sciences, 116, 11866-11871.

415 Muller, H. J. (1918). Genetic variability, twin hybrids and constant hybrids, in a case of

416 balanced lethal factors. Genetics, 3, 422-499.

417 Mullon, C., Wright, A. E., Reuter, M., Pomiankowski, A., \& Mank, J. E. (2017). Evolution of 418 dosage compensation under sexual selection differs between $\mathrm{X}$ and $\mathrm{Z}$ chromosomes. Nature 419 Communications, 6, 7720.

420 Naurin, S., Hasselquist, D., Bensch, S., \& Hansson, B. (2012). Sex-biased gene expression on 421 the avian $\mathrm{Z}$ chromosome: highly expressed genes show higher male-biased expression. PloS 422 One, 7, e46854.

423 Nielsen, S. V., Guzmán-Méndez, I. A., Gamble, T., Blumer, M., Pinto, B. J., Kratochvíl, L., 424 \& Rovatsos, M. Escaping the evolutionary trap? Sex chromosome turnover in basilisks and 425 related lizards (Corytophanidae: Squamata). Biology Letters, 15, 20190498.

426 O’Connor, R. E., Romanov, M. N., Kiazim, L. G., Barrett, P. M., Farré, M., Damas, J., ... 427 Griffin, D. K. (2018). Reconstruction of the diapsid ancestral genome permits chromosome 428 evolution tracing in avian and non-avian dinosaurs. Nature Communications, 9, 1883.

429 Ohno, S. (1967). Sex chromosomes and sex-linked genes. Berlin, Heidelberg, New York: 430 Springer-Verlag. 
431 Pan, Q., Anderson, J., Bertho, S., Herpin, A., Wilson, C., Postlethwait, J. H., ... Y. Guiguena.

432 (2019). Vertebrate sex-determining genes play musical chairs. Comptes Rendus Biologies, $433339,258-262$.

434 Pessia, E., Engelstädter, J., \& Marais, G. A. (2013). The evolution of X chromosome 435 inactivation in mammals: the demise of Ohno's hypothesis? Cellular and Molecular Life $436 \quad$ Sciences, $71,1383-1394$.

437 Picard, M. A. L., Cosseau, C., Ferré, S., Quack, T., Grevelding, C. G., Couté, Y., \& Vicoso, 438 B. (2018). Evolution of gene dosage on the Z-chromosome of schistosome parasites. eLife, 7, $439 \mathrm{e} 35684$.

440 Rice, A. M, \& McLysaght, A. (2017). Dosage-sensitive genes in evolution and disease. BMC 441 Biology, 15, 78.

442 Rovatsos, M., Pokorná, M., Altmanová, M., \& Kratochvíl, L. (2014a). Cretaceous park of sex 443 determination: sex chromosomes are conserved across iguanas. Biology Letters, 10, 44420131093.

445 Rovatsos, M., Altmanová, M., Johnson Pokorná, M., \& Kratochvíl, L. Novel X-linked genes 446 revealed by quantitative polymerase chain reaction in the green anole, Anolis carolinensis. 447 G3-Genes Genome Genetics, 4, 2107-2113.

448 Rovatsos, M., Vukić, J., \& Kratochvíl, L. (2016). Mammalian X homolog acts as sex 449 chromosome in lacertid lizards. Heredity.;117:8-13.

450 Rovatsos, M., Vukić, J., Altmanová, M., Johnson Pokorná, M., Moravec, J., \& Kratochvíl L. 451 (2016). Conservation of sex chromosomes in lacertid lizards. Molecular Ecology, 25, 31204523126.

453 Rovatsos, M., Praschag, P., Fritz, U., \& Kratochvíl, L. (2017). Stable Cretaceous sex 454 chromosomes enable molecular sexing in softshell turtles (Testudines: Trionychidae). 455 Scientific Reports, 7, 42150. 
456 Rovatsos, M., Augstenová, B., Altmanová, M., Sloboda, M., Kodym, P., \& Kratochvíl, L.

457 (2018). Triploid colubrid snake provides insight into the mechanism of sex determination in 458 advanced snakes. Sexual Development, 12, 251-255.

459 Rovatsos, M., Rehák, I., Velenský, P., \& Kratochvíl, L. (2019). Shared ancient sex

460 chromosomes in varanids, beaded lizards and alligator lizards. Molecular Biology and 461 Evolution, 36, 1113-1120.

462 Rovatsos, M., Gamble T., Nielsen S. V., Georges A., Ezaz T., Kratochvíl, L. (2020). Do male 463 and female heterogamety really differ in expression regulation? Lack of global dosage 464 balance in pygopodid geckos. Philosophical Transactions of the Royal Society B: Biological 465 Sciences, in press. (doi: https://doi.org/10.1101/2020.06.03.132241)Rupp, S. M., Webster, T.

466 H., Olney, K. C., Hutchins, E. D., Kusumi, K., \& Wilson Sayres, M. A. (2017). Evolution of 467 dosage compensation in Anolis carolinensis, a reptile with XX/XY chromosomal sex 468 determination. Genome Biology and Evolution, 9, 231-240.

469 Smith, C. A., Roeszler, K. N., Ohnesorg, T., Cummins, D. M., Farlie, P. G., Doran, T. J., \& 470 Sinclair, A. H. The avian Z-linked gene DMRT1 is required for male sex determination in the 471 chicken. Nature, 461, 267-271.

472 Vicoso, B, \& Bachtrog, D. (2009). Progress and prospects toward our understanding of the 473 evolution of dosage compensation. Chromosome Research, 17, 585.

474 Vicoso, B., Emerson, J. J., Zektser, Y., Mahajan, S., \& Bachtrog, D. (2013). Comparative sex 475 chromosome genomics in snakes: differentiation, evolutionary strata, and lack of global 476 dosage compensation. PLoS Biology, 11, e1001643.

477 Wang, Z., Pascual-Anaya, J., Zadissa, A., Li, W., Niimura, Y., Huang, Z., ... Kirie, N. (2013). 478 The draft genomes of soft-shell turtle and green sea turtle yield insights into the development 479 and evolution of the turtle-specific body plan. Nature Genetics, 45, 701-706. 
480 Wilson Sayres, M. A., \& Makova, K. D. (2011). Genome analyses substantiate male mutation

481 bias in many species. BioEssays, 33, 938-945.

482 Zanetti, S., \& Puoti, A. (2013). Sex determination in the Caenorhabditis elegans germline. Pp

483 41-69 in: Schedl, T. (ed.) Germ Cell Development in C. elegans. Advances in Experimental

484 Medicine and Biology, vol. 757. Springer, New York.

485 Zhang, Y., \& Oliver, B. (2007). Dosage compensation goes global. Current Opinion in 486 Genetics \& Development, 17, 113-120.

487 Zimmer, F., Harrison, P. W., Dessimoz, C., \& Mank, J. E. (2016). Compensation of dosagesensitive genes on the chicken Z chromosome. Genome Biology and Evolution, 8, 1233-1242. 


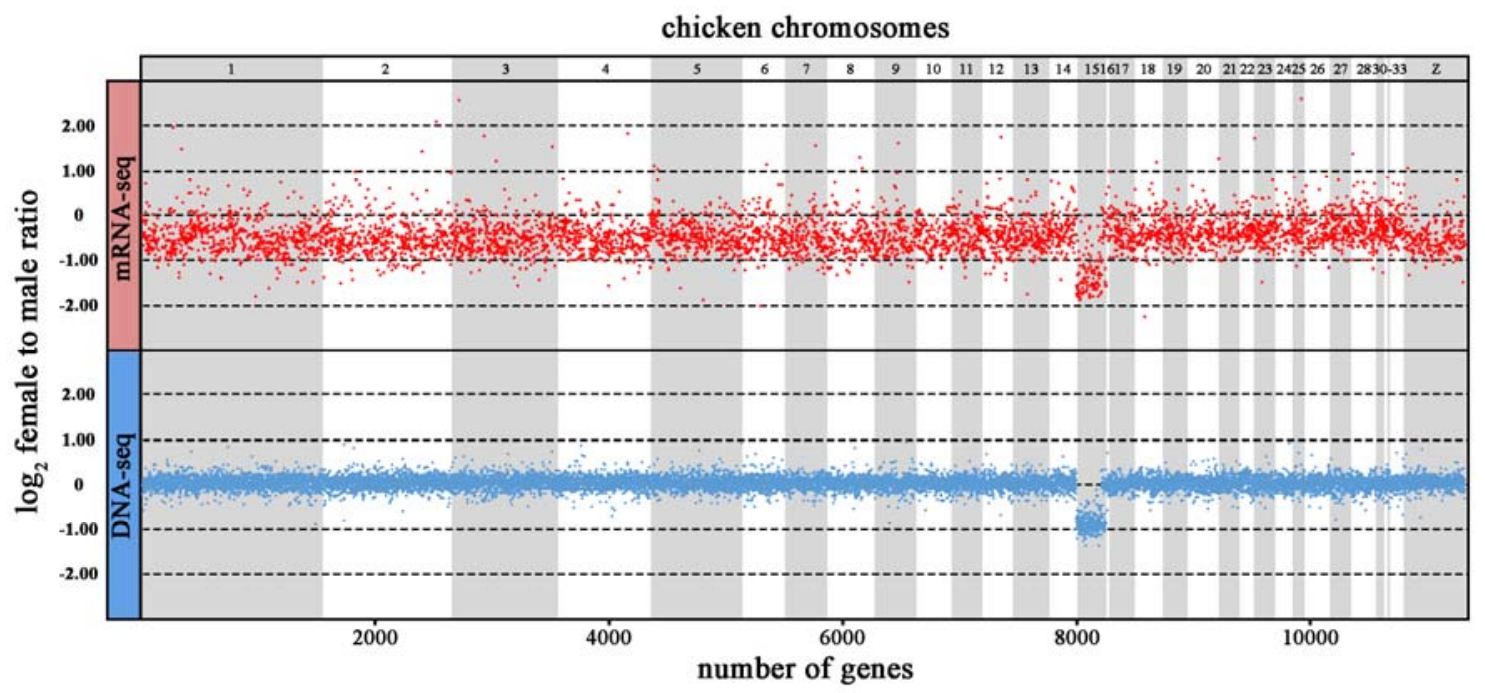

492 Fig. 1: $\log _{2}$-transformed female to male ratios in DNA-seq read coverage (blue) and in expression (RPKM, red) across identified genes of Apalone ferox. Each dot corresponds to the $\mathrm{f} / \mathrm{m}$ ratio from a gene. In the absence of a chromosome-level genome assembly in trionychid turtles, the genes are illustrated according to the position of their orthologs in the chicken genome. Note that the region homologous to chicken chromosome 15 possess much lower ratios in both read coverage depth and RPKM, demonstrating that this part of genome is Zspecific and lacks dosage balance in expression between sexes in most genes in the turtle. 


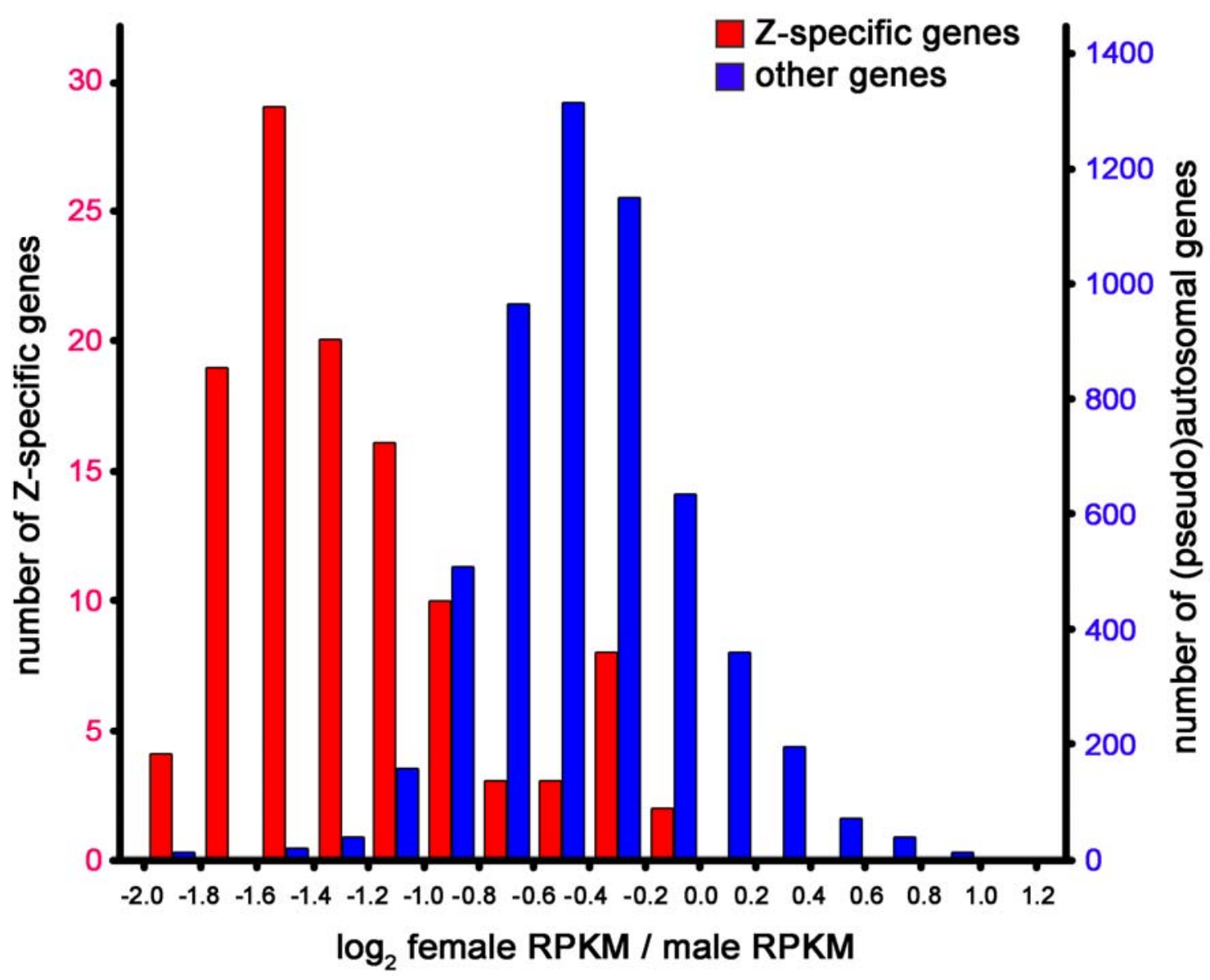

Fig. 2: Histogram of the $\log _{2}$-transformed female to male ratios in the expression measure 


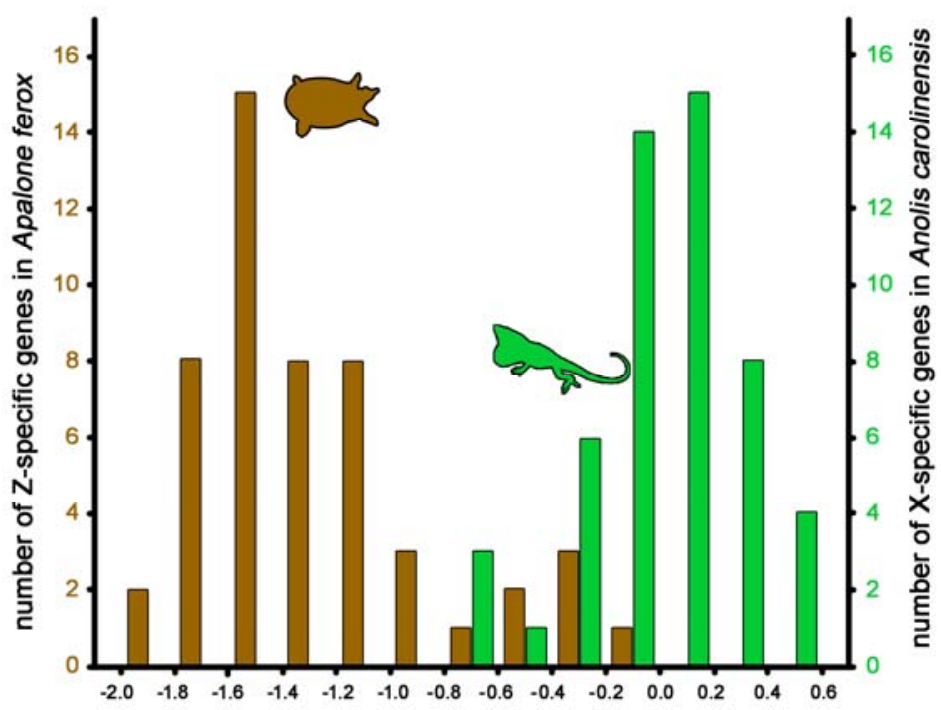

a.

$\log _{2}$ female RPKM / male RPKM

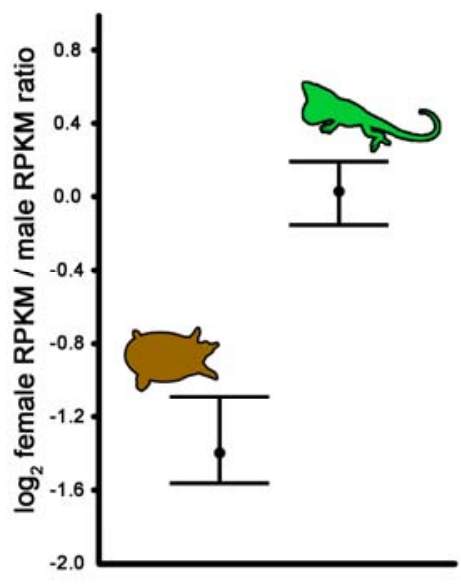

b.

521 Fig. 3: Comparison of the sexual differences in expression of the orthologous genes which are shows mostly dosage balance in the expression between sexes in the lizard but not in the

524 turtle. Histograms (a) and medians and $25^{\text {th }}$ and $75^{\text {th }}$ quartiles (b) of female to male ratios in 525 RPKM are given.

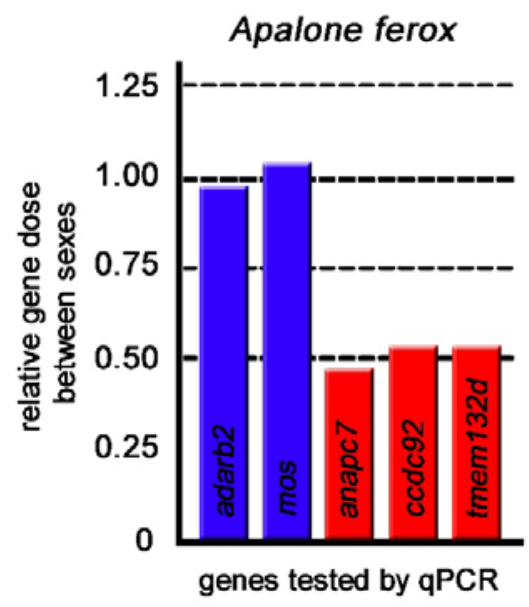

528 Fig. S1: Relative gene dose ratios (r) between females and males for each primer pair for autosomal control (blue) and Z-specific genes (red) in three pairs of Apalone ferox. The gene ragl was used for normalization of the qPCR values. 


\section{Supplementary information}

532

533 Table S1: Parameters for mapping Illumina mRNA-seq reads in reference transcripts in 534 Geneious v. R7.1.

535

536 Table S2: List of examined genes from the genome of Apalone ferox and the position of their

537 homologous genes to chicken (Gallus gallus). Female to male (f/m) ratios are presented for 538 both DNA-seq read coverage analysis and RPKM expression values in A. ferox.

539

540 Table S3: List of 45 orthologous genes which are X-specific in A. carolinensis and Z-specific

541 in A. ferox. Data for A. carolinensis were collected from Marin et al. (2017) and Rupp et al. 542 (2017). 